\title{
NASH in Lean Individuals
}

\author{
Ramy Younes, $\mathrm{MD}^{1} \quad$ Elisabetta Bugianesi, $\mathrm{MD}, \mathrm{PhD}^{1}$ \\ 1 Department of Medical Sciences, Division of Gastroenterology, \\ University of Torino, AOU Città della Salute e della Scienza, Torino, \\ Italy \\ Address for correspondence Prof. Elisabetta Bugianesi, MD, PhD, \\ Department of Medical Sciences, University of Torino, AOU Città della \\ Salute e della Scienza, Corso Dogliotti 14, 10126 Torino, Italy \\ Semin Liver Dis 2019;39:86-95. \\ (e-mail: elisabetta.bugianesi@unito.it).
}

\begin{abstract}
Keywords

- nonalcoholic fatty liver disease

- body mass index

- visceral adiposity

- insulin resistance

- genetic polymorphism

- ethnicity

Nonalcoholic fatty liver disease (NAFLD) is generally associated with obesity and the related comorbidities but it can also develop in subjects with a body mass index (BMI) within the ethnic-specific cutoff of $25 \mathrm{~kg} / \mathrm{m}^{2}$ BMI in Caucasian and $23 \mathrm{~kg} / \mathrm{m}^{2}$ in Asian subjects, the so-called "lean" NAFLD. This sub-phenotype of NAFLD patients has been described across populations of different ethnicity, particularly in Asia, but it can be diagnosed in 10 to $20 \%$ of nonobese Americans and Caucasians. Pathophysiological mechanisms underpinning the "lean" phenotype are not completely understood, but they may include a more dysfunctional fat (visceral obesity, differences in adipocyte differentiation and altered lipid turnover), altered body composition (decreased muscle mass), a genetic background, not limited to patatin-like phospholipase domain-containing protein 3 (PNPLA3) C > G polymorphisms, epigenetic changes occurring early in life and a different pattern of gut microbiota. Lean subjects with NAFLD have milder features of the metabolic syndrome when compared with obese patients. Nonetheless they have a higher prevalence of metabolic alterations (e.g., dyslipidemia, arterial hypertension, insulin resistance, and diabetes) compared with healthy controls. Data on histological severity are controversial, but they can develop the full spectrum of liver disease associated with nonalcoholic steatohepatitis NASH. Since lean NAFLD usually present with less obesity-related comorbidities, it is commonly believed that this group would follow a relatively benign clinical course but recent data challenge this concept. Here, the authors describe the current knowledge about NAFLD in lean individuals and highlight the unanswered questions and gaps in the field.
\end{abstract}

Nonalcoholic fatty liver disease (NAFLD) is rapidly becoming the most frequent cause of liver disease in Western countries, with an almost exponential increase in South America, Asia, and the Middle East. From its early description, we know that NAFLD is intimately connected with obesity, type 2 diabetes (T2DM), and the metabolic syndrome (MetS), and therefore its spread in parallel with the worldwide pandemic of obesity is not surprising. Nevertheless, NAFLD can develop in the absence of obesity, the so-called "lean" NAFLD (i.e., body mass index [BMI] within the ethnic-specific cutoff of $25 \mathrm{~kg} /$ $\mathrm{m}^{2}$ in Caucasian and $23 \mathrm{~kg} / \mathrm{m}^{2}$ in Asian subjects). By this definition, NAFLD in subjects with normal BMI has been frequently described in the Asian population, but it can be diagnosed in 10 to $20 \%$ of nonobese Americans and Caucasians. ${ }^{1,2}$ Despite the apparent "healthier" phenotype, these patients may display the whole spectrum of the histopathological features of nonalcoholic steatohepatitis (NASH), that is, steatosis, lobular inflammation, hepatocyte ballooning, and/or fibrosis. $^{3}$ Overall, many clinicians have a perception of lean NAFLD being more "benign" in nature, but recent data challenge this view and suggest that it should not be overlooked.

It is conceivable that lean NAFLD comprises an heterogeneous spectrum of different causes ( - Table $\mathbf{1}$ ), ranging from environmental cases (such as high fructose and high fat published online January 17, 2019
Copyright $\odot 2019$ by Thieme Medical Publishers, Inc., 333 Seventh Avenue, New York, NY 10001, USA. Tel: +1(212) 584-4662. ISSN 0272-8087. 
Table 1 Possible causes of nonalcoholic fatty liver disease in lean subjects

\begin{tabular}{|l|}
\hline High fructose intake \\
\hline Metabolically obese normal-weight subjects \\
\hline Congenital and acquired (highly active antiretroviral therapy for HIV) lipodistrophy \\
\hline Malnutrition (Kwashiorkor) \\
\hline Genetic \\
Patatin-like phospholipase domain-containing protein 3 (PNPLA 3) GG variant \\
Congenital defects of metabolism \\
$\quad$ - Familial Hypobetalipoproteinemia (FHBL) \\
$\quad$ - Lysosomal acid lipase deficiency (LAL-D) \\
\hline Endocrine disorders (polycystic ovary syndrome, hypothyroidism, or growth hormone deficiency) \\
\hline Drug-related (amiodarone, methotrexate, and tamoxifen) \\
\hline Jejunoileal bypass, starvation, total parenteral nutrition \\
\hline
\end{tabular}

intake), body fat distribution (visceral obesity as opposed to general obesity), body composition (acquired or congenital lipodistrophy, sarcopenia) and genetic risk factors, including rare congenital defects of metabolism such as lysosomal acid lipase deficiency (LAL-D) and familial hypobetalipoprotein B (FHLB). While the description of secondary causes of lean NAFLD is beyond the scope of this review, we will describe the current knowledge about NAFLD in lean individuals and highlight the unanswered questions and gaps in the field.

\section{Epidemiology}

Epidemiological studies about prevalence, incidence, and natural history of NAFLD and NASH in lean subjects suffer from several limitations: on one hand, the "classic" bias of the criteria adopted to define NAFLD in the general population (liver function tests vs. imaging vs. algorithms) and the lack of noninvasive markers for NASH; on the other hand, the heterogeneous definition of lean across studies. In fact, while most of these studies compared patients using 25 as BMI cutoff, in Asian cohorts the term lean should be used according to the ethnic-specific BMI cutoff of 23, while patients with $\mathrm{BMI}<25 \mathrm{~kg} / \mathrm{m}^{2}$ should be commonly indicated as "nonobese." ${ }^{4}$ In this regard, interpreting BMI values with the simplistic association between low BMI and low body fat is misleading because BMI is an imperfect index of adiposity, particularly in truncal fat accumulation, and does not take into account body composition. Adding to the confusion is that the definition of MetS, commonly used to detect metabolic abnormalities, varies across studies and even more at the individual level, so that the association between the degree of obesity and development of insulin resistance may not be so clear-cut.

Population studies had being describing NAFLD in nonobese subjects since 2006 (- Table 2). In a study including 2520 residents of the Shengang Township in Taiwan, NAFLD (by abdominal ultrasound) was found in 61 over 1,444 nonobese participants (4.2\%), in the absence of other etiologies of chronic liver disease. ${ }^{5}$ In a prospective epidemiological study carried in a very poor, rural area of West Bengal, India, NAFLD was identified in $8.6 \%$ of the overall population, but $75 \%$ of NAFLD subjects belonged to the nonobese group. ${ }^{6}$ The nonobese and lean individuals with NAFLD had more subcutaneous fat, higher fasting blood glucose, and higher levels of triglycerides. However, this population also included $47 \%$ with malnutrition, which can be associated with NAFLD by a different mechanism (choline deficiency).

After these two pioneer epidemiological surveys, most of the studies investigating the nonobese pattern of NAFLD had been performed in the Asian continent. In China, the Zhejiang Zhenhai Study evaluated the prevalence and risk factors of NAFLD in 6,905 nonobese individuals (BMI $<25 \mathrm{~kg} / \mathrm{m}^{2}$ ). NAFLD was diagnosed by ultrasound in $7.27 \%$ of the study participants. ${ }^{7}$ Similarly, in a cohort of 2,000 Chinese subjects who received annual physical examinations, NAFLD was found in nearly $18 \%$ of the nonobese subjects (BMI $<24$ $\left.\mathrm{kg} / \mathrm{m}^{2}\right){ }^{8}$ A large Korean population study recruiting 29,994 individuals who presented for a routine health evaluation, reported a prevalence of NAFLD of $12.6 \%$ in the nonobese participants $(n=3,014) .{ }^{9}$ These findings were confirmed in another Korean general medical check-up program where $22.4 \%$ of nonobese subjects (333 of 1,487 ) had NAFLD. ${ }^{10}$ In Japan, the overall prevalence of NAFLD in 3,271 subjects who received health checkups from 2011 to 2012 was 68.5\% in obese subjects and $15.2 \%$ in nonobese subjects. Metabolic factors such as waist circumference and triglycerides were predictors of nonobese NAFLD. Interestingly, weight gain since early adulthood (around the age of 20) was significantly associated with NAFLD in nonobese subjects of both genders. ${ }^{11}$ In a community-based Hong-Kong cohort, NAFLD was detected by proton-magnetic resonance spectroscopy (1HMRS) in $19.3 \%$ of the lean cases (BMI $<23 \mathrm{~kg} / \mathrm{m}^{2}$ ), compared with $61 \%$ in those with a higher BMI. ${ }^{12}$

Compared with the Asian surveys described earlier, studies in the Caucasian population are less numerous and, in general, involved a smaller number of subjects. In Italy, the Dionysus Study showed that the prevalence of lean NAFLD $\left(\mathrm{BMI}<25 \mathrm{~kg} / \mathrm{m}^{2}\right.$ ) assessed by ultrasonography was $16 \%$, compared with $75.8 \%$ in obese. ${ }^{13}$ In Iceland, the AGESReykjavik Study Investigators highlighted an association between the central axes of obesity with the presence of MetS in lean patients. ${ }^{14}$ In this study, CT scan showed the 
Table 2 Main epidemiological, cross-sectional, and longitudinal studies describing the characteristics of lean patients in NAFLD

\begin{tabular}{|c|c|c|}
\hline \multicolumn{3}{|l|}{ Epidemiological Studies } \\
\hline & Main findings ${ }^{a}$ & Ethnicity \\
\hline Chen et al, $2006^{5}$ & $\begin{array}{l}2520 \text { Taiwanese subjects included. NAFLD was found in } 4.2 \% \text { of nonobese } \\
\text { participants. }\end{array}$ & Asian \\
\hline Das $\mathrm{K}$ et al, $2010^{6}$ & $\begin{array}{l}\text { Of the } 1911 \text { individuals included, NAFLD was found in } 8.6 \% \text { of the population, of } \\
\text { whom } 75 \% \text { were nonobese. }\end{array}$ & Indian \\
\hline Kwon et al, $2012^{9}$ & $\begin{array}{l}\text { Large Korean population study }(n=29944) \text {. NAFLD reported a prevalence of } 12.6 \% \\
\text { in the nonobese participants. }\end{array}$ & Asian \\
\hline Kim et al, $2011^{14}$ & $\begin{array}{l}\text { Icelandic study including } 2945 \text { patients. Hepatic steatosis was shown even in } \\
\text { patients with a median BMI of } 22.7 \mathrm{~kg} / \mathrm{m}^{2}\end{array}$ & Caucasian \\
\hline Browning et al, $2004^{15}$ & $\begin{array}{l}\text { In the Dallas Heart Study cohort, the prevalence of steatosis in subjects with } \\
\mathrm{BMI}<30 \mathrm{~kg} / \mathrm{m}^{2} \text { was } 17 \%\end{array}$ & Caucasian \\
\hline Younossi et al, $2012^{16}$ & $\begin{array}{l}\text { Among the } 11613 \text { subjects of the NHANES III cohort, the prevalence of NAFLD in lean } \\
\text { individuals was } 7.4 \%\end{array}$ & Caucasian \\
\hline \multicolumn{3}{|l|}{ Cross-sectional Studies } \\
\hline C. Selvakumar, $2018^{17}$ & $\begin{array}{l}\text { Among } 1482 \text { U.S. lean adolescence, } 8 \% \text { had NAFLD, and had a higher prevalence of } \\
\text { low HDL, hypertriglyceridemia and insulin resistance. }\end{array}$ & Caucasian \\
\hline Younossi et al, $2012^{16}$ & $\begin{array}{l}\text { Lean NAFLD was associated with younger age, female sex, insulin resistance, and } \\
\text { hypercholesterolemia }\end{array}$ & Caucasian \\
\hline Feldman et al, $2017^{46}$ & $\begin{array}{l}\text { Although lean NAFLD patients have a better metabolic profile, they have lower } \\
\text { adiponectin levels when compared with healthy controls. }\end{array}$ & Caucasian \\
\hline Feng et al, $2014^{8}$ & Lean NAFLD have a more active and proinflammatory visceral adipose tissue & Asian \\
\hline Wong et al, $2015^{31}$ & $\begin{array}{l}\text { Waist circumference and triglyceride levels predicted the incidence of steatosis in } \\
\text { lean individuals }\end{array}$ & Asian \\
\hline Fracanzani et al, $2017^{30}$ & $\begin{array}{l}\text { Lean patients had a lower rate of MetS, diabetes, lower cardiovascular damage, and } \\
\text { lower hepatic histological damage }\end{array}$ & Caucasian \\
\hline Akyuz et al, $2015^{52}$ & $\begin{array}{l}\text { Turkish cohort with lean prevalence of } 7.6 \% \text {. High hemoglobin levels were the only } \\
\text { independent predictor of NASH and fibrosis in lean subjects. }\end{array}$ & Caucasian \\
\hline \multicolumn{3}{|l|}{ Longitudinal Studies } \\
\hline Leung et al, $2017^{3}$ & $\begin{array}{l}\text { Median follow-up of 49-months. Hypertriglyceridemia and higher creatinine were } \\
\text { associated with advanced liver disease in lean. Death and HCC were recorded only in } \\
\text { the obese group. }\end{array}$ & Asian \\
\hline Cruz et al, $2014^{55}$ & $\begin{array}{l}\text { Despite a better metabolic profile, less insulin resistance, and fibrosis, lean subjects } \\
\text { have a higher overall mortality than patients with NAFLD who are overweight or } \\
\text { obese. }\end{array}$ & Mixed \\
\hline Hagstrom et al, $2018^{56}$ & $\begin{array}{l}\text { After a median follow-up of } 19.9 \text { years, although patients with lean NAFLD showed } \\
\text { lower fibrosis, they were at higher risk for development of severe liver disease } \\
\text { compared with patients with NAFLD and a higher BMI, independent of available } \\
\text { confounders. }\end{array}$ & Caucasian \\
\hline
\end{tabular}

Abbreviations: BMI, body mass index; HCC, hepatocellular carcinoma; HDL, high-density lipoprotein; NAFLD, nonalcoholic fatty liver disease; NHANES, Third National Health and Nutrition Examination Survey.

an all studies, alternative etiologies of liver disease has been excluded.

presence of hepatic steatosis even in patients with a median BMI of $22.7 \mathrm{~kg} / \mathrm{m}^{2}$. In the Dallas Heart Study, the prevalence of steatosis (defined as a hepatic triglyceride content $>5.5 \%$ by $1 \mathrm{H}-\mathrm{MRS}$ ) in subjects with a BMI $<30 \mathrm{~kg} / \mathrm{m}^{2}$ was $17 \%$, compared with $34 \%$ in the overall study subjects. ${ }^{15}$ The prevalence of NAFLD was significantly lower in nonobese African Americans (11\%) but comparable in nonobese Caucasians and nonobese Hispanics (20\% vs. $26 \% ; p=0.12$ ). ${ }^{15}$ However, the largest epidemiological study analyzing prevalence and features of lean Caucasian NAFLD patients has been carried in the United States, using data of the Third
National Health and Nutrition Examination Survey III (NHANES III). ${ }^{16}$ Among 11,613 subjects, the prevalence of NAFLD at ultrasonography was as high as $27.7 \%$ in overweight/obese subjects, significantly higher than the $7.4 \%$ observed in subjects with normal BMI $\left(<25 \mathrm{~kg} / \mathrm{m}^{2}\right)$.

The presence of a lean phenotype with NAFLD is observed across all age groups, including adolescents. Cross-sectional data from 1,482 lean subjects (BMI $<85$ th percentile) aged between 12 and 18 years, enrolled in the NHANES during the 2005 to 2014 cycles, reported a $8 \%$ prevalence of suspected NAFLD (by ALT above the low gender-specific cutoff) among 
lean adolescents. Low HDL, hypertriglyceridemia, and presence of insulin resistance were more common among lean NAFLD compared with their non-NAFLD counterparts. ${ }^{17}$

Overall, epidemiologic data suggest that the prevalence of lean NAFLD is approximately 5 to $26 \%$ in the Asian population and 7 to $20 \%$ in the Western one. This suggests that factors independent from body weight may be important in a subset of NAFLD subjects.

\section{Pathophysiology of Lean NAFLD}

Pathologic pathways underlying the development of NAFLD in lean subjects are not entirely understood. However, studies on adipose tissue functions, genetic analyses, studies in vitro and in vivo on animal models, and finally gut microbiome research can provide some hints to mechanisms (-Fig. 1).

\section{Insulin Resistance and Fat Distribution}

Despite a reduced likelihood of being associated with the components of MetS, subjects with lean NAFLD are nonetheless insulin resistant when compared with healthy controls. In a cohort of subjects with biopsy-proven NAFLD, free of diabetes, obesity, and MetS, the metabolic pattern of insulin resistance in the main target tissues (muscle, liver, and adipose tissue) was similar to that observed in obesity, with adipose tissue insulin resistance playing an important role despite a low BMI and normal subcutaneous fat. ${ }^{18}$ This early finding was further supported by more recent studies, showing higher circulating concentration of free fatty acids (FFA) in lean NAFLD patients compared with healthy controls and a higher portal FFA flow, which may induce intrahepatic fat accumulation. ${ }^{19,20}$

It is likely that a vast part of lean NAFLD belong to the phenotype of "metabolically obese normal weight" (MONW) subjects, described in at least $5 \%$ of the occidental population, who display altered insulin sensitivity and increased cardiovascular risk. ${ }^{21}$ When comparing metabolically healthy and unhealthy normal weight subjects, the latter population has increased liver fat content, visceral fat mass, and carotid intima media thickness (cIMT), but lower subcutaneous fat mass, insulin sensitivity, and insulin secretion. ${ }^{22}$ These key characteristic of MONW are consistent across studies in humans; increased liver fat is probably due to a decreased capacity for storing fat in subcutaneous adipose tissue, coupled with reduced mitochondrial function and increased de novo lipogenesis in the liver. ${ }^{22}$ Furthermore, MONW subjects also have a proinflammatory circulating milieu characterized by decreased adiponectin concentration and a unique $\mathrm{T}$ cell signature. ${ }^{21}$ Ethnicity has a significant impact on the variability of MONW across studies. In the Multiethnic Study of Atherosclerosis, the prevalence of metabolically unhealthy with normal weight ranged from $21 \%$ in whites, $32 \%$ in Chinese Americans, $31 \%$ in

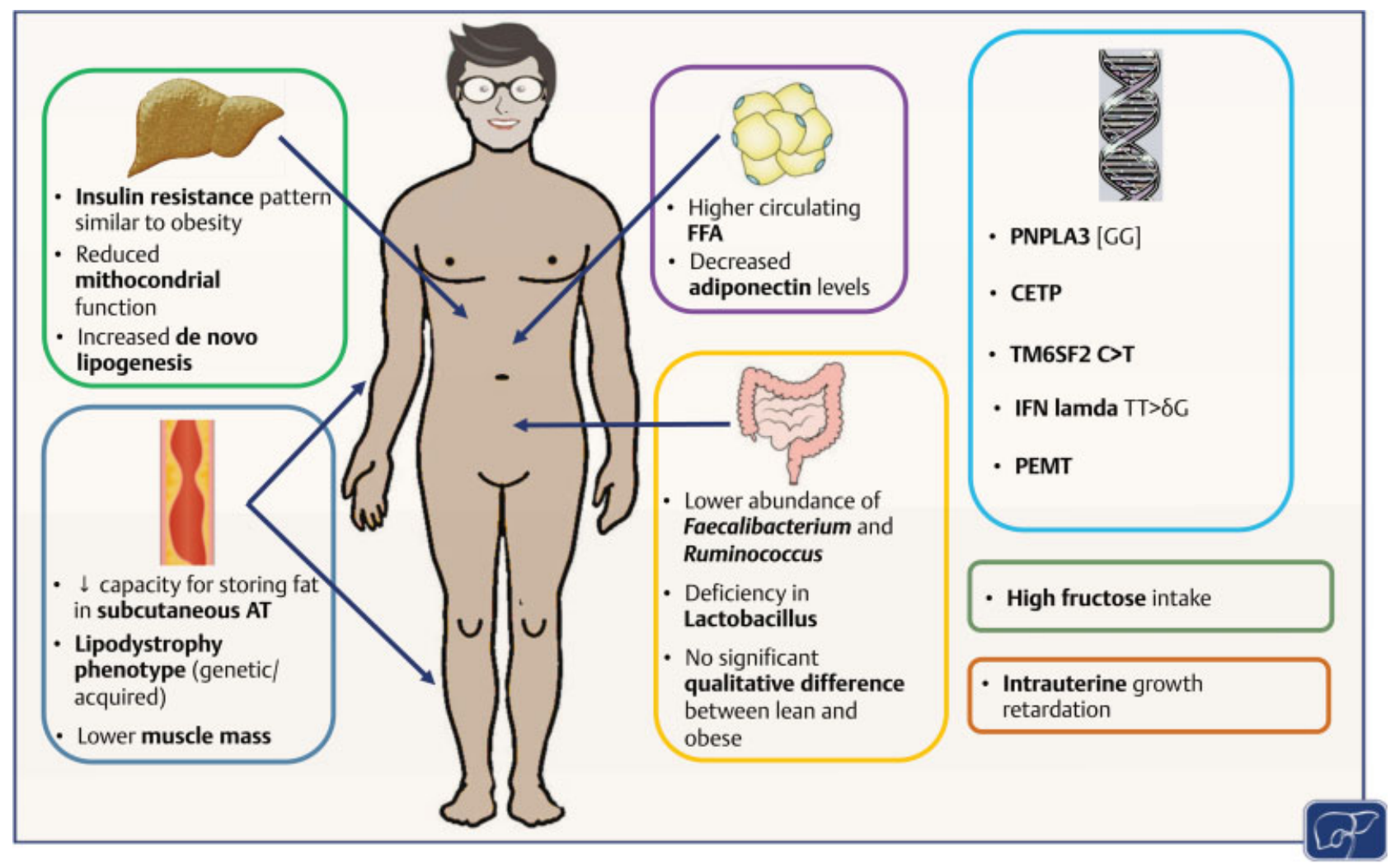

Fig. 1 Pathophysiological determinants of nonalcoholic fatty liver disease in lean subjects. AT indicates adipose tissue; FFA, free fatty acids; PNPLA3, patatin-like phospholipase domain-containing protein 3; TM6SF2, transmembrane 6 superfamily member 2; CETP, cholesteryl ester transfer protein; IFN, interferon; PEMT, phosphatidylethanolamine N-methyltransferase. 
African Americans, 38.5\% in Hispanics, and $43.6 \%$ in South Asians. ${ }^{23}$ In the same study, the prevalence of nonobese NAFLD (BMI $<30 \mathrm{~kg} / \mathrm{m}^{2}$ ) assessed by CT scan was $11 \%$, including 9\% among Caucasians, 6\% among African Americans, and $18 \%$ among Hispanic Americans. ${ }^{23}$

At the extreme end of the spectrum of MONW lays the lipodystrophy phenotype. These subjects typically display absence of fat in the classic subcutaneous depots but large ectopic accumulation of lipids in the skeletal muscle and in the liver associated with severe insulin resistance. ${ }^{24}$ While the genetic forms are relatively rare, acquired lipodystrophy can be found in HIV patients under highly active antiretroviral therapy (HAART) therapy and can be also found in some lean people not having been diagnosed with lipodystrophy.

The importance of body composition in the onset and progression of NAFLD is also supported by the finding that sarcopenia, defined as a progressive and generalized loss of skeletal muscle mass, strength, and function, is a novel risk factor for the development of NAFLD. ${ }^{25}$ Recently, we found elevated plasma amino acid concentrations in NAFLD subjects either with or without obesity, likely to be related to peripheral resistance and resulting in increased muscle proteolysis during the fasting state, which lends support to the pathogenesis of sarcopenia in NAFLD. ${ }^{26}$

\section{Genetic and Environmental Factors}

The data discussed earlier suggest that lean NAFLD have an increased susceptibility to develop NAFLD that can be partially attributed to genetic factors or epigenetic changes induced early in life.

The search for genetic causes that contribute to the incidence of NAFLD in lean patients is still in its infancy. As reported by genome-wide association analyses, first performed within the Dallas Heart Study and widely confirmed in literature, a single variant in the patatin-like phospholipase domain-containing protein 3 (PNPLA3), the rs738409[G], encoding I148M, is associated with an increase in both liver fat and hepatic inflammation. ${ }^{27}$ Comparing obese and nonobese subjects with NAFLD, the prevalence of the PNPLA3 [G] allele was significantly higher among nonobese individuals (78.4\% vs. $59.8 \%$ ) and was independently associated with NAFLD even in the multivariate analysis. ${ }^{12} \mathrm{~A}$ study recruiting lean Japanese NAFLD patients (BMI $<23 \mathrm{~kg} / \mathrm{m}^{2}$ ) confirmed the previous findings. Although there were no GG homozygous carriers in their population, in the lean group the $G$ allele was a predictor of NAFLD in all multivariate analysis steps, while it was not in the obese population. ${ }^{28}$ Another Japanese study recruiting 540 biopsy-proven NAFLD patients (134 nonobese) found a higher rs738409 GG homozygous genotype rate in nonobese NAFLD patients compared with obese individuals with fatty liver. Again, the GG-single-nucleotide polymorphism was an independent predictor of NAFLD, together with diabetes, in the nonobese cohort only. ${ }^{29}$ In a retrospective study on patients with lean NAFLD, the only variable associated independently with NASH and a fibrosis score of 2 or higher was rs738409 $\mathrm{C}>\mathrm{G}$ in PNPLA3 ${ }^{30}$ However, in a prospective general population study in Hong Kong including 565 cases (BMI $<23 \mathrm{~kg} / \mathrm{m}^{2}$ in $72 \%$ ) without evidence of NAFLD (by 1H-MRS) at baseline, the presence of the common variant in the PNPLA3 gene did not provide any relevant clue for incident NAFLD. ${ }^{31}$

Other variants in different loci may be involved in individual cases. Cholesteryl ester transfer protein (CETP) is involved in triglyceride exchange between lipoproteins. ${ }^{32}$ Two single-nucleotide polymorphisms (rs12447924 and rs12597002) were associated with fatty liver disease in adolescent lean Caucasian females (BMI $<25 \mathrm{~kg} / \mathrm{m}^{2}$ ). The highest risk of NAFLD was found in the group with the lowest adiposity assessed by skinfold thickness, where the prevalence of NAFLD in lean homozygotes, heterozygotes, and wild type was more than $30 \%, 10$ to $15 \%$ and $3.5 \%$ respectively. ${ }^{33} \mathrm{~A}$ single-nucleotide polymorphism in transmembrane 6 superfamily member 2 (TM6SF2) was associated with NAFLD and fibrosis independent of age, diabetes, obesity, and the PNPLA3 genotype. ${ }^{34}$ In a retrospective cohort, a significantly greater proportion of patients with lean NAFLD carried rs58542926 C > T in TM6SF2 (4\%) than obese or overweight individuals. ${ }^{30}$ The interferon (IFN) lambda 4 rs368234815 TT $>\delta G$ variant, influencing innate immunity regulation, has been linked to liver damage in patients with NAFLD. The impact of rs368234815 seems generally more marked in nonobese individuals, where an association with severe fibrosis, necroinflammation, and NASH has been observed. ${ }^{35}$

Finally, a recent study on animal models hypothesized that a deficiency of the phosphatidylethanolamine $\mathrm{N}$ methyltransferase (PEMT) could play a key role in the development of NASH in lean individuals. ${ }^{36}$ PEMT is an enzyme involved in the synthesis of phosphatidylcholines in liver cells. PEMT - / - mice on high fat-high sucrose diet did not develop obesity or insulin resistance compared with the PEMT $+/+$ and presented normal cholesterol and triglyceride levels. Nonetheless, PEMT $-\mid-$ developed NASH and after 90 weeks all PEMT -/- mice developed liver tumors. When PEMT mRNA expression in human liver biopsies was quantified, a lower expression of PEMT was found in patients with NASH. A correlation with lower BMI has been also reported, suggesting that PEMT deficiency could be an etiologic agent of lean NASH. ${ }^{36}$

Insulin resistance in adipose tissue develops early during fetal growth restriction and is maintained during the neonatal period and adulthood. ${ }^{37}$ Besides genetic factors, intrauterine growth might play a role in favoring NAFLD, particularly in children. An Italian group first described the association of intrauterine growth retardation with pediatric NAFLD and more severe disease activity at histology, independent of and in addition to insulin resistance. At an average age of 11 years, most study subjects (80\%) were insulin resistant, despite normal BMI and a very low prevalence of metabolic abnormalities. Notably, the family history of type 2 diabetes was less common, suggesting that genetic factors have lower relevance in the onset of NAFLD in this cohort. ${ }^{38}$

The setting of a smaller adipocyte number during early life let lean population mostly change their adipocyte volume in adulthood, developing MetS features at a lower fat mass, 39 partially explaining why these subjects easily develop NAFLD 
for small increases in body weight, still in the nonobese range. $^{31}$

Finally, among the most common environmental causes, a high fructose intake is an additional risk factor for NAFLD and NASH, particularly in children and adolescents. In a study on young nonobese subjects without obvious metabolic risk factors, the only independent predictor for the presence of NAFLD was a higher soft drink and juices consumption, up to fourfold compared with healthy controls. ${ }^{40}$ Thus, preventing fructose intake may represent a readily modifiable environmental factor, particularly in younger lean NAFLD patients.

\section{Gut Microbiome and Metabolomics}

Fatty liver has been associated with a lower rate of Bacteroidetes and a higher rate of Prevotella and Porphyromonas, as well as a higher number of ethanol-producing bacteria. ${ }^{41}$ Duarte et al described a significant difference in the abundance of Faecalibacterium, Ruminococcus, Lactobacillus, and Bifidobacterium in patients with NASH when compared with controls. ${ }^{42}$ The subgroup of lean patients with NASH had had less abundance of Ruminococcus and a deficiency of Lactobacilli when compared with overweight and obese patients with NASH. ${ }^{43}$

Lyso-phosphatidylcholines (lyso-PCs) are phospholipids with anti-inflammatory and insulin-sensitizer effects and lower levels of lyso-PCs are observed in obesity. ${ }^{42,44,45}$ Metabolomic analyses demonstrated lower levels of lysoPCs in both lean and obese NAFLD patients when compared with healthy controls. ${ }^{46}$ On the other hand, when compared their obese counterpart, lean patients with NAFLD showed a higher level of lysine concentration. ${ }^{46}$ Being related to visceral fat accumulation, ${ }^{47}$ lysine may represent a sign of the dysfunctional metabolic environment underpinning lean NAFLD individuals.

\section{Clinical and Pathological Features}

Compared with their healthy counterpart, lean NAFLD have a reduced likelihood of being associated with the components of MetS; nonetheless subjects have an increased prevalence of metabolic derangements, above all diabetes and higher plasma triglycerides, although both abnormalities are usually less severe than in obese NAFLD patients. ${ }^{16,48}$ In the NHANES III population, when compared with overweight/obese NAFLD subjects, lean NAFLD was independently associated with younger age, female sex, insulin resistance, and hypercholesterolemia. Among individuals who fulfilled the clinical definition of NASH, as it was adapted for that cohort (i.e., NAFLD patients with elevated aminotransferases in the presence of either diabetes or insulin resistance), the prevalence of lean subjects was as low as $1.38 \%{ }^{16}$ Another study in Caucasians confirmed that patients with lean NAFLD have a better metabolic profile than overweight and obese, that is, higher levels of highdensity lipoprotein cholesterol (HDL-C), lower triglyceride, and fasting glucose levels, in addition to a lower concentration of the proinflammatory cytokines Interleukin 6 and tumor necrosis factor alpha. In addition, leptin levels were similar to healthy controls and significantly lower than in obese NAFLD patients. Conversely, in lean NAFLD adiponectin levels have been found significantly lower than healthy controls, but similar to the obese NAFLD. ${ }^{46}$ In Asian cohorts, features of MetS have been associated with the development of NAFLD across the entire BMI spectrum, but lean NAFLD patients presented the strongest correlations. ${ }^{7,8}$ Furthermore, lean NAFLD seems to have a more active visceral adipose tissue, in terms of visceral adiposity index (an indicator of visceral fat function associated with cardiometabolic risk), when compared with overweight or obese population. ${ }^{8,49}$ Noteworthy, in some lean healthy subjects, relatively small changes in their metabolic profile and body weight can be associated with incident lean NAFLD. A prospective cohort study from Hong Kong included a subgroup of 406 lean (BMI $<23.0 \mathrm{~kg} / \mathrm{m}^{2}$ ) subjects, of whom $7.9 \%$ developed incident steatosis (by $1 \mathrm{H}-\mathrm{MRS}$ ) after an interval of 3 to 5 years (median: 47 months). ${ }^{31}$ At multivariable analysis, increased waist circumference and serum triglyceride levels during follow-up were associated with incident fatty liver, although some of these patients did not develop a full MetS.

Even though a better metabolic profile is supposed to be associated with a less severe histological damage, there is no agreement on this issue and some studies suggest the contrary in lean NAFLD. A seminal Italian study ${ }^{50}$ reported the presence of NASH in $50 \%$ of lean NAFLD patients (BMI $<25$ $\mathrm{kg} / \mathrm{m}^{2}$ ). The prevalence of NASH was quite similar across BMI classes (normal weight, 65\%; overweight, 73\%; and obese, $84 \% ; p=0.184$ ). Another study, including 430 biopsy-proven NAFLD, showed that $55 \%$ of patients without visceral obesity according to waist circumference had NASH and fibrosis $\geq$ F2, despite milder metabolic alterations. ${ }^{51}$ On the contrary, in another retrospective series including 669 biopsy-proven Caucasian NAFLD patients, when compared with overweight and obese patients, NAFLD subjects with a BMI $<25 \mathrm{~kg} / \mathrm{m}^{2}$ had a lower rate of MetS and diabetes, lower cardiovascular damage, expressed as cIMT, and prevalence of carotid plaques as well as lower prevalence of histologically diagnosed NASH and fibrosis F2 or higher. ${ }^{30}$ Of interest, in a Turkish cohort of 483 biopsy-proven NAFLD patients, with a prevalence of lean NAFLD (BMI $<25 \mathrm{~kg} / \mathrm{m}^{2}$ ) of $7.6 \%$, high hemoglobin levels was the only independent predictor of NASH and advanced fibrosis in lean individuals and not in the obese/overweight group. ${ }^{52}$

In a study from China, similar proportions of obese and nonobese patients had NASH (51.9 vs. $43.5 \%, p=0.217$ ), although the latter ones had a lower degree of steatosis and hepatocyte ballooning, and the proportion of patients with advanced fibrosis was not different in the two groups. ${ }^{3}$ Triglyceride levels independently predicted disease severity in nonobese NAFLD group and were associated with both higher grade of steatosis and hepatocyte ballooning. ${ }^{3}$ Similarly, Kumar et al found no difference in NASH prevalence between lean $\left(B M I<23 \mathrm{~kg} / \mathrm{m}^{2}\right)$ and non-lean subjects among 110 biopsy-proven NAFLD patients of Indian ethnicity. ${ }^{53}$ In a Japanese cohort of biopsy-proven NAFLD patients, lobular inflammation, hepatocyte ballooning, and NAFLD activity score were strictly associated with the GG-PNPLA3 
single-nucleotide polymorphism, which was more prevalent in the nonobese cohort and was not associated with histological severity in NAFLD obese patients. ${ }^{29}$

Thus, lean NAFLD patients may present milder histological features or may show the same characteristics when compared with obese patients with NAFLD, but overall they can display the full spectrum of liver damage. This suggests that the risk of cirrhosis for lean NAFLD patients may not be that different after all, and that, once NASH is established, obesity may not be the main driver of fibrosis progression. The next important question is whether lean NAFLD subjects have an adverse outcome related to liver-related morbidity and mortality.

\section{Outcome and Prognosis of Lean NAFLD patients}

Since lean NAFLD usually present with less obesity-related comorbidities, it is commonly believed that this group would follow a relatively benign clinical course. Within the cohort of the NHANES III, ${ }^{54}$ mortality of metabolically normal NAFLD patients was similar to the cohort without liver disease. However, the longitudinal risk of mortality in lean NAFLD has been scarcely explored. In the Hong Kong cohort of Leung et al, where 307 patients with biopsy-proven NAFLD (23.5\% nonobese, BMI $<25 \mathrm{~kg} / \mathrm{m}^{2}$ ) had been followed up for a median period of 49 months, clinical events similarly occurred in obese (11.9\%) and nonobese patients (8.3\%). Cardiovascular morbidity accounted for about two-thirds of all major events in both groups. All deaths $(n=6)$ occurred in the obese group, but definite conclusions are difficult to make as follow-up was relatively short. ${ }^{3}$ An international study with a longer follow-up period, published so far only in abstract form, challenged the concept that the prognosis of patients with NAFLD who have normal $\mathrm{BMI}$ is better than in those who are overweight or obese. ${ }^{55}$ In a total of 1090 prospectively recruited patients, 125 (11.5\%) were classified as lean (BMI $<25 \mathrm{~kg} / \mathrm{m}^{2}$ for non-Asians and $<23 \mathrm{~kg} / \mathrm{m}^{2}$ for Asians). Lean NAFLD were more commonly men of non-Caucasian origin and, as expected, showed less features of the MetS. Histologically, they had less severe fibrosis but a higher grade of lobular inflammation. Interestingly, median survival free of liver transplantation was significantly shorter in lean than in non-lean patients (18.1 vs. 26.6 years, respectively, $p<0.001$ ). The higher risk of death/liver transplantation in lean NAFLD was independent of the classic risk factors that may influence the development of this outcome.

In another retrospective study including 646 patients with biopsy-proven NAFLD, at enrolment in NAFLD, $19 \%$ of patients were lean, $52 \%$ overweight, and $29 \%$ obese. Patients with lean NAFLD were older, had lower transaminases, lower stages of fibrosis, and lower prevalence of NASH compared with patients with a higher BMI. During a mean follow-up of 19.9 years (range: $0.4-40$ years) patients with lean NAFLD had no increased risk for overall mortality, but they were more likely to develop severe liver disease than patients with NAFLD who were overweight (hazard ratio: $2.69 ; p=0.007$ ), independent of available confounders. ${ }^{56}$ Three prognostic indicators for mortality in lean NAFLD were identified: older age, fibrosis stage, and hypertension. Noteworthy, of the 19 patients with lean NAFLD who developed severe liver disease, $58 \%(n=11)$ had fibrosis of stage 0 to 2 at baseline. $A$ limitation of the longitudinal retrospective studies is the limited ability to determine whether or not subjects developed additional risk factors over time that are known to predispose to advanced liver disease, such as diabetes or changes in alcohol intake and body weight.

Certainly future longitudinal prospective studies are needed to define the prognosis in lean NAFLD and to elucidate potential pathophysiologic mechanisms underlying progression; nevertheless data available suggest that carefully selected patients with lean NAFLD likely require longterm follow-up and reassessment of progression of liver disease over time.

\section{Management}

When it comes to the prevention and treatment of lean NAFLD, current European guideline states that follow-up is mandatory in obesity, but it also suggests follow-up in lean persons with NAFLD because of possible disease progression, even though they have less severe metabolic disturbance (B2 strength of evidence $)^{57}$. Careful identification and correction of environmental causes, such as significant fructose consumption, may be effective particularly in young patients. General recommendations include an adoption of a healthy lifestyle and the initiation of pharmacological treatments for elevated blood pressure, dyslipidemia, and hyperglycemia, if necessary. ${ }^{57}$ However, these guidelines do not provide much information as to whether and to what extent prevention and treatment should be adapted in lean patients, given the harder correction of underlying risk factors. Weight loss remains the background therapy in all cases with overweight/obesity, but in lean NAFLD the efficacy of calorie restriction has not been tested. Nevertheless, diet should be prescribed when a weight gain even within the nonobese BMI range was preceding the development of lean NAFLD. Importantly, habitual physical activity should certainly be indicated because it can specifically decrease visceral fat. ${ }^{58}$ Treatment with the thiazolidinedione pioglitazone also reduced the diabetes risk and improved insulin secretion in nonobese subjects with impaired glucose tolerance, ${ }^{59}$ but no analysis had been performed in normal-weight individuals. Incretin-based treatments may be more effective in overweight and obese subjects than in normal-weight individuals because its efficacy is associated with weight loss. ${ }^{60}$ In the Liraglutide and Cardiovascular Outcomes in Type 2 Diabetes (LEADER) study, which included 9,340 patients, treatment with liraglutide was associated with lower incidence of the primary composite outcome in obese patients with type 2 diabetes, but not in nonobese. ${ }^{61}$

\section{Conclusion}

Lean patients with NAFLD are a well-defined entity and are described by numerous studies both in the Eastern continent and in the Western world. Considering that lean NAFLD patients can develop the full spectrum of liver damage that 
characterize non-lean NAFLD, it is important to understand what phenotypes characterize this population. Compared with healthy individuals, lean subjects with NAFLD present metabolic risk factors (dyslipidemia, arterial hypertension, diabetes, and insulin resistance) to a significantly greater extent, probably due to a more dysfunctional adipose tissue, not limited to its visceral component. Although literature data indicate that these patients have more favorable metabolic characteristics when compared with obese NAFLD patients, data on long-term survival and mortality are insufficient and controversial. Furthermore, genetic analyses suggest that metabolic risk appears to be determined by different pathways in normal-weight and obese subjects and indicates that the genetic background could be the key to better characterize this type of patients. These findings may have several implications for clinical interventions and for drug development. Applying well-defined phenotyping strategies in clinical trials to separate the outcome in lean and obese NAFLD subjects will help to more precisely understand the pathophysiology of liver disease. Without a doubt, the challenges that the lean NAFLD raises are an excellent incentive for the development of future research.

\section{Main Concepts and Learning Points}

- The presence of NAFLD in subjects with a BMI within the ethnic-specific cutoff of $25 \mathrm{~kg} / \mathrm{m}^{2}$ in Caucasian and $23 \mathrm{~kg} / \mathrm{m}^{2}$ in Asian has been defined as lean NAFLD.

- Lean NAFLD has been initially described in the Asian population; it can be diagnosed in approximately 5 to $26 \%$ of the general population in Asia and 7 to $20 \%$ in the Western world.

- Pathophysiological mechanisms are not totally understood and may include a dysfunctional adipose tissue, altered body composition, genetic mutations, epigenetic changes occurring early in life and a different pattern of gut microbiota.

- Although this phenotype has generally a more favorable metabolic profile when compared with obese NAFLD, lean NAFLD patients can develop the full spectrum of liver damage that characterizes non-lean NAFLD.

- Data on long-term prognosis of lean patients are insufficient and controversial but suggest that lean NAFLD is not a "benign" disease

- General recommendations include an adoption of a healthy lifestyle, but guidelines do not provide much information as to whether and to what extent prevention and treatment should be adapted in lean patients, given the harder correction of underlying risk factors.

\section{Acknowledgments}

The study was supported by grants for EPoS (Elucidating Pathways of Steatohepatitis, Grant Agreement No:634413) and for LITMUS (Liver Investigation: Testing Marker Utility in Steatohepatitis, Grant Agreement No: 777377) projects, funded by the Horizon 2020 Framework Program of the European Union.

\section{References}

1 Younossi Z, Anstee QM, Marietti M, et al. Global burden of NAFLD and NASH: trends, predictions, risk factors and prevention. Nat Rev Gastroenterol Hepatol 2018;15(01):11-20

2 Ruderman NB, Schneider SH, Berchtold P. The "metabolicallyobese," normal-weight individual. Am J Clin Nutr 1981;34(08): $1617-1621$

3 Leung JC, Loong TC, Wei JL, et al. Histological severity and clinical outcomes of nonalcoholic fatty liver disease in nonobese patients. Hepatology 2017;65(01):54-64

4 Consultation WHOE; WHO Expert Consultation. Appropriate body-mass index for Asian populations and its implications for policy and intervention strategies. Lancet 2004;363 (9403):157-163

5 Chen $\mathrm{CH}$, Huang MH, Yang JC, et al. Prevalence and risk factors of nonalcoholic fatty liver disease in an adult population of Taiwan: metabolic significance of nonalcoholic fatty liver disease in nonobese adults. J Clin Gastroenterol 2006;40(08):745-752

6 Das K, Das K, Mukherjee PS, et al. Nonobese population in a developing country has a high prevalence of nonalcoholic fatty liver and significant liver disease. Hepatology 2010;51(05): 1593-1602

7 Xu C, Yu C, Ma H, Xu L, Miao M, Li Y. Prevalence and risk factors for the development of nonalcoholic fatty liver disease in a nonobese Chinese population: the Zhejiang Zhenhai Study. Am J Gastroenterol 2013;108(08):1299-1304

8 Feng RN, Du SS, Wang C, et al. Lean-non-alcoholic fatty liver disease increases risk for metabolic disorders in a normal weight Chinese population. World J Gastroenterol 2014;20(47): 17932-17940

9 Kwon YM, Oh SW, Hwang SS, Lee C, Kwon H, Chung GE. Association of nonalcoholic fatty liver disease with components of metabolic syndrome according to body mass index in Korean adults. Am J Gastroenterol 2012;107(12):1852-1858

$10 \mathrm{Kim} \mathrm{NH}$, Kim JH, Kim YJ, et al. Clinical and metabolic factors associated with development and regression of nonalcoholic fatty liver disease in nonobese subjects. Liver Int 2014;34(04):604-611

11 Nishioji K, Sumida Y, Kamaguchi M, et al. Prevalence of and risk factors for non-alcoholic fatty liver disease in a non-obese Japanese population, 2011-2012. J Gastroenterol 2015;50(01):95-108

12 Wei JL, Leung JC, Loong TC, et al. Prevalence and severity of nonalcoholic fatty liver disease in non-obese patients: a population study using proton-magnetic resonance spectroscopy. Am J Gastroenterol 2015;110(09):1306-1314, quiz 1315

13 Bellentani S, Saccoccio G, Masutti F, et al. Prevalence of and risk factors for hepatic steatosis in Northern Italy. Ann Intern Med 2000;132(02):112-117

14 Kim LJ, Nalls MA, Eiriksdottir G, et al; AGES-Reykjavik Study Investigators. Associations of visceral and liver fat with the metabolic syndrome across the spectrum of obesity: the AGES-Reykjavik study. Obesity (Silver Spring) 2011;19(06): 1265-1271

15 Browning JD, Szczepaniak LS, Dobbins R, et al. Prevalence of hepatic steatosis in an urban population in the United States: impact of ethnicity. Hepatology 2004;40(06):1387-1395

16 Younossi ZM, Stepanova M, Negro F, et al. Nonalcoholic fatty liver disease in lean individuals in the United States. Medicine (Baltimore) 2012;91(06):319-327

17 Conjeevaram Selvakumar PK, Kabbany MN, Lopez R, Rayas MS, Lynch JL, Alkhouri N. Prevalence of suspected nonalcoholic fatty liver disease in lean adolescents in the United States. J Pediatr Gastroenterol Nutr 2018;67(01):75-79

18 Bugianesi E, Gastaldelli A, Vanni E, et al. Insulin resistance in nondiabetic patients with non-alcoholic fatty liver disease: sites and mechanisms. Diabetologia 2005;48(04):634-642

19 Feng R, Luo C, Li C, et al. Free fatty acids profile among lean, overweight and obese non-alcoholic fatty liver disease patients: a case-control study. Lipids Health Dis 2017;16(01):165 
20 Wattacheril J, Sanyal AJ. Lean NAFLD: an underrecognized outlier. Curr Hepatol Rep 2016;15(02):134-139

21 Conus F, Rabasa-Lhoret R, Péronnet F. Characteristics of metabolically obese normal-weight (MONW) subjects. Appl Physiol Nutr Metab 2007;32(01):4-12

22 Stefan N, Schick F, Häring HU. Causes, characteristics, and consequences of metabolically unhealthy normal weight in humans. Cell Metab 2017;26(02):292-300

23 Gujral UP, Vittinghoff E, Mongraw-Chaffin M, et al. Cardiometabolic abnormalities among normal-weight persons from five racial/ethnic groups in the United States: a cross-sectional analysis of two cohort studies. Ann Intern Med 2017;166(09): 628-636

24 Hussain I, Garg A. Lipodystrophy syndromes. Endocrinol Metab Clin North Am 2016;45(04):783-797

25 Bhanji RA, Narayanan P, Allen AM, Malhi H, Watt KD. Sarcopenia in hiding: the risk and consequence of underestimating muscle dysfunction in nonalcoholic steatohepatitis. Hepatology 2017;66 (06):2055-2065

26 Gaggini M, Carli F, Rosso C, et al. Altered amino acid concentrations in NAFLD: Impact of obesity and insulin resistance. Hepatology 2018;67(01):145-158

27 Romeo S, Kozlitina J, Xing C, et al. Genetic variation in PNPLA3 confers susceptibility to nonalcoholic fatty liver disease. Nat Genet 2008;40(12):1461-1465

28 Nishioji K, Mochizuki N, Kobayashi M, et al. The impact of PNPLA3 rs738409 genetic polymorphism and weight gain $\geq 10 \mathrm{~kg}$ after age 20 on non-alcoholic fatty liver disease in non-obese Japanese individuals. PLoS One 2015;10(10):e0140427

29 Honda Y, Yoneda M, Kessoku T, et al. Characteristics of non-obese non-alcoholic fatty liver disease: effect of genetic and environmental factors. Hepatol Res 2016;46(10):1011-1018

30 Fracanzani AL, Petta S, Lombardi R, et al. Liver and cardiovascular damage in patients with lean nonalcoholic fatty liver disease, and association with visceral obesity. Clin Gastroenterol Hepatol 2017;15(10):1604-1611.e1

31 Wong VW, Wong GL, Yeung DK, et al. Incidence of non-alcoholic fatty liver disease in Hong Kong: a population study with paired proton-magnetic resonance spectroscopy. J Hepatol 2015;62(01): 182-189

32 Watts GF, Riches FM, Humphries SE, Talmud PJ, van Bockxmeer FM. Genotypic associations of the hepatic secretion of VLDL apolipoprotein B-100 in obesity. J Lipid Res 2000;41(03):481-488

33 Adams LA, Marsh JA, Ayonrinde OT, et al. Cholesteryl ester transfer protein gene polymorphisms increase the risk of fatty liver in females independent of adiposity. J Gastroenterol Hepatol 2012;27(09):1520-1527

34 Liu YL, Reeves HL, Burt AD, et al. TM6SF2 rs58542926 influences hepatic fibrosis progression in patients with non-alcoholic fatty liver disease. Nat Commun 2014;5:4309

35 Petta S, Valenti L, Tuttolomondo A, et al. Interferon lambda 4 rs368234815 TT $>\delta G$ variant is associated with liver damage in patients with nonalcoholic fatty liver disease. Hepatology 2017; 66(06):1885-1893

36 Nakatsuka A, Matsuyama M, Yamaguchi S, et al. Insufficiency of phosphatidylethanolamine $\mathrm{N}$-methyltransferase is risk for lean non-alcoholic steatohepatitis. Sci Rep 2016;6:21721

37 Spalding KL, Arner E, Westermark PO, et al. Dynamics of fat cell turnover in humans. Nature 2008;453(7196):783-787

38 Nobili V, Marcellini M, Marchesini G, et al. Intrauterine growth retardation, insulin resistance, and nonalcoholic fatty liver disease in children. Diabetes Care 2007;30(10):2638-2640

39 Das K, Chowdhury A. Lean NASH: distinctiveness and clinical implication. Hepatol Int 2013;7(Suppl 2):806-813

40 Abid A, Taha O, Nseir W, Farah R, Grosovski M, Assy N. Soft drink consumption is associated with fatty liver disease independent of metabolic syndrome. J Hepatol 2009;51(05):918-924
41 Zhu L, Baker SS, Gill C, et al. Characterization of gut microbiomes in nonalcoholic steatohepatitis (NASH) patients: a connection between endogenous alcohol and NASH. Hepatology 2013;57 (02):601-609

42 Soga T, Ohishi T, Matsui T, et al. Lysophosphatidylcholine enhances glucose-dependent insulin secretion via an orphan $\mathrm{G}$ protein-coupled receptor. Biochem Biophys Res Commun 2005; 326(04):744-751

43 Duarte SMB, Stefano JT, Miele L, et al. Gut microbiome composition in lean patients with NASH is associated with liver damage independent of caloric intake: A prospective pilot study. Nutr Metab Cardiovasc Dis 2018;28(04):369-384

44 Hung ND, Kim MR, Sok DE. 2-Polyunsaturated acyl lysophosphatidylethanolamine attenuates inflammatory response in zymosan A-induced peritonitis in mice. Lipids 2011;46(10): 893-906

45 Heimerl S, Fischer M, Baessler A, et al. Alterations of plasma lysophosphatidylcholine species in obesity and weight loss. PLoS One 2014;9(10):e111348

46 Feldman A, Eder SK, Felder TK, et al. Clinical and metabolic characterization of lean Caucasian subjects with non-alcoholic fatty liver. Am J Gastroenterol 2017;112(01):102-110

47 Yamakado M, Tanaka T, Nagao K, et al. Plasma amino acid profile is associated with visceral fat accumulation in obese Japanese subjects. Clin Obes 2012;2(1-2):29-40

48 Vos B, Moreno C, Nagy N, et al. Lean non-alcoholic fatty liver disease (Lean-NAFLD): a major cause of cryptogenic liver disease. Acta Gastroenterol Belg 2011;74(03):389-394

49 Amato MC, Giordano C, Galia M, et al; AlkaMeSy Study Group. Visceral Adiposity Index: a reliable indicator of visceral fat function associated with cardiometabolic risk. Diabetes Care 2010;33 (04):920-922

50 Marchesini G, Bugianesi E, Forlani G, et al. Nonalcoholic fatty liver, steatohepatitis, and the metabolic syndrome. Hepatology 2003; 37(04):917-923

51 Fracanzani AL, Valenti L, Bugianesi E, et al. Risk of nonalcoholic steatohepatitis and fibrosis in patients with nonalcoholic fatty liver disease and low visceral adiposity. J Hepatol 2011;54(06): 1244-1249

52 Akyuz U, Yesil A, Yilmaz Y. Characterization of lean patients with nonalcoholic fatty liver disease: potential role of high hemoglobin levels. Scand J Gastroenterol 2015;50(03):341-346

53 Kumar R, Rastogi A, Sharma MK, et al. Clinicopathological characteristics and metabolic profiles of non-alcoholic fatty liver disease in Indian patients with normal body mass index: do they differ from obese or overweight non-alcoholic fatty liver disease? Indian J Endocrinol Metab 2013;17(04):665-671

54 Younossi ZM, Otgonsuren M, Venkatesan C, Mishra A. In patients with non-alcoholic fatty liver disease, metabolically abnormal individuals are at a higher risk for mortality while metabolically normal individuals are not. Metabolism 2013;62(03):352-360

55 Cruz ACD, Bugianesi E, George J, et al. 379 Characteristics and long-term prognosis of lean patients with nonalcoholic fatty liver disease. Gastroenterology 2014;146(05):S-909

56 Hagström H, Nasr P, Ekstedt M, et al. Risk for development of severe liver disease in lean patients with nonalcoholic fatty liver disease: a long-term follow-up study. Hepatol Commun 2017;2 (01):48-57

57 European Association for the Study of the Liver (EASL); European Association for the Study of Diabetes (EASD); European Association for the Study of Obesity (EASO). EASL-EASD-EASO Clinical Practice Guidelines for the management of non-alcoholic fatty liver disease. J Hepatol 2016;64(06):1388-1402

58 Ohkawara K, Tanaka S, Miyachi M, Ishikawa-Takata K, Tabata I. A dose-response relation between aerobic exercise and visceral fat reduction: systematic review of clinical trials. Int J Obes 2007;31 (12):1786-1797 
59 DeFronzo RA, Tripathy D, Schwenke DC, et al; ACT NOW Study. Pioglitazone for diabetes prevention in impaired glucose tolerance. N Engl J Med 2011;364(12):1104-1115

60 Armstrong MJ, Gaunt P, Aithal GP, et al; LEAN trial team. Liraglutide safety and efficacy in patients with nonalcoholic steatohepatitis (LEAN): a multicentre, double-blind, randomised, placebo-controlled phase 2 study. Lancet 2016;387 (10019):679-690

61 Marso SP, Daniels GH, Brown-Frandsen K, et al; LEADER Steering Committee; LEADER Trial Investigators. Liraglutide and cardiovascular outcomes in type 2 diabetes. N Engl J Med 2016;375(04): $311-322$ 